\title{
Asymptotic properties of coverings in negative curvature
}

\author{
ANDREA SAMBUSETti
}

\begin{abstract}
We show that the universal covering $\tilde{X}$ of any compact, negatively curved manifold $X_{0}$ has an exponential growth rate which is strictly greater than the exponential growth rate of any other normal covering $X \rightarrow X_{0}$. Moreover, we give an explicit formula estimating the difference between $\omega(\tilde{X})$ and $\omega(X)$ in terms of the systole of $X$ and of other elementary geometric parameters of the base space $X_{0}$. Then we discuss some applications of this formula to periodic geodesics, to the bottom of the spectrum and to the critical exponent of normal coverings.
\end{abstract}

53C23; 53C21, 53C22, 20F67, 20F69

\section{Introduction}

In this paper we shall investigate an asymptotic property of normal coverings of compact, negatively curved manifolds, and some of its consequences. A qualitative version of this property, known as growth tightness, can be stated as follows. Recall that the growth function of a complete Riemannian manifold $X$ is the function $v_{X}(x, R)$ given by the volume of balls of radius $R$ of $X$ centered at some point $x$, and that its exponential growth rate is defined as

$$
\omega(X)=\limsup _{R \rightarrow \infty} R^{-1} \cdot \log v_{X}(x, R)
$$

(this is clearly independent of the choice of the base point $x$, as well as all the asymptotic properties of the function $v_{X}$ ).

Theorem 1 Let $X_{0}$ be a closed $n$-dimensional negatively curved manifold. For any Riemannian normal covering $X$ of $X_{0}$, different from the universal covering $\tilde{X}$, we have $\omega(\tilde{X})>\omega(X)$.

Notice that the inequality $\omega(\tilde{X}) \geq \omega(X)$ is obvious, as $R$-balls of $\tilde{X}$ always have volume greater or equal than the corresponding balls of $X$; Theorem 1 says that the universal covering of a closed negatively curved manifold $X_{0}$ is characterized as the normal covering of maximal exponential growth rate. Any Riemannian manifold 
$X_{0}$ having this property will be called growth tight. We call the difference $\Omega(X)=$ $\omega(\tilde{X})-\omega(X)$ the asymptotic deficit of the Riemannian manifold $X$. We shall give later a formula estimating $\Omega(X)$ for a non-simply connected normal covering $X$.

The above result generalizes our results in [10], where we proved growth tightness of hyperbolic surfaces, by using Gauss-Bonnet's formula and elementary topology of dimension 2. Remark that this strict inequality is peculiar of negative curvature, and it does not hold in general, even for nonpositively curved manifolds of exponential growth. Consider for instance the Riemannian product $X_{0}=\Sigma_{1} \times \Sigma_{g}$ of a flat torus with any closed hyperbolic surface. Then $\tilde{X}$ has exponential growth and nonpositive sectional curvature, but the normal covering $X=\Sigma_{1} \times \widetilde{\Sigma}_{g}=\Sigma_{1} \times \mathbb{H}^{2} \rightarrow X_{0}$ has the same exponential growth as $\tilde{X}$ (as the fiber of $\tilde{X} \rightarrow X$ is an orbit of the abelian group $\pi_{1}\left(\Sigma_{1}\right)$ and has polynomial growth in $\left.\tilde{X}\right)$.

The notion of "growth tightness" was originally introduced by Grigorchuk and de la Harpe [6] for finitely generated groups endowed with a word metric. Actually, a discrete group $(G, d)$ endowed with a left-invariant distance is growth tight if $\omega(G, d)>\omega(G / N, d / N)$ for every nontrivial normal subgroup $N$ (where $\omega(G, d)=$ $\limsup _{R \rightarrow \infty} R^{-1} \cdot \log \# B_{(G, d)}(e, R)$ is the exponential growth rate of balls of $G$ with respect to $d$, and $d / N$ is the distance induced on the quotient group $G / N$ ). Arzhantseva and Lysenok [1] proved that every Gromov hyperbolic group without finite normal subgroups (in particular, the fundamental group of any closed, negatively curved manifold) is growth tight with respect to word metrics. Our Theorem 1 is the geometric analogue of Arzhantseva and Lysenok's result, as it can be group-theoretically reformulated by saying that the fundamental group $\pi_{1}\left(X_{0}\right)$ of any compact negatively curved manifold is growth tight with respect to the geometric distance $d$ induced by the Riemannian length $d s^{2}$, that is, when we define $d\left(g_{1}, g_{2}\right)$ as the Riemannian length of the smallest geodesic loop, at some fixed base point, representing $g_{1}^{-1} g_{2}$ (see the beginning of Section 5). However, it should be noticed that our result is independent from Arzhantseva and Lysenok's; in fact, the geometric distance on $\pi_{1}\left(X_{0}\right)$ induced by the Riemannian metric is not a word metric and, even if quasi-isometric to any word metric, the exponential growth rate of $\pi_{1}\left(X_{0}\right)$ (hence, apriori, growth tightness) is not invariant under quasi-isometries.

Actually our proof is conceptually very different from that in [1], as we do not deal with counting words, nor do we have an efficient algorithm as a finite automaton in the Riemannian framework. We construct, from any nontrivial quotient $\Gamma=G / N$ of $G=\pi_{1}\left(X_{0}\right)$, a new discrete metric space that we call $\ell_{c}^{1}(\Gamma)$, which is made of finite, arbitrarily long sequences of elements of $\Gamma$, and we show that it can be immersed into $G$ by a map which contracts distances. This will immediately give that $\omega(G) \geq \omega\left(\ell_{c}^{1}(\Gamma)\right)$, while a direct computation (Proposition 21) will show that $\omega\left(\ell_{c}^{1}(\Gamma)\right)>\omega(\Gamma)$ (actually, 
this argument will not be applied directly to $\Gamma$, but to some maximal, $\delta$-separated net of $\Gamma$ ). Our method to construct the contracting immersion $j: \ell_{c}^{1}(\Gamma) \hookrightarrow G$ consists in lifting a sequence of elements $\left(\gamma_{i}\right)$ of $\Gamma$ to $G$ and then enchain them in a particular way, so that the knowledge of $j\left(\gamma_{1}, \ldots, \gamma_{m}\right)$ permits us to recover the whole sequence of $\gamma_{i}$ 's; to describe properly this process of enchaining, we define a new operation (the "twisted product") on the isometry group of $\tilde{X}$, whose main properties are studied in Section 3.

We shall now discuss some consequences of Theorem 1.

Let us consider the problem of estimating the systole of a (possibly open) non-simply connected Riemannian manifold $X$. Recall that the systole of $X$, denoted sys $(X)$, is the length of the shortest noncontractible closed curve of $X$ (the systole may of course vanish for noncompact manifolds, eg a hyperbolic manifold with some cusp, whereas it equals $+\infty$ by definition if $X$ is simply connected). The value of the systole can theoretically be detected by the difference between the growth functions of $\tilde{X}$ and $X$. Actually, if $R_{0}$ is the first point where they separate, that is,

$$
R_{0}=\inf \left\{R \mid v_{\tilde{X}}(\tilde{x}, R)>v_{X}(x, R), \tilde{x} \in \tilde{X}, x \text { projection of } \tilde{x} \text { to } X\right\}
$$

then it is easy to show that sys $(X)=2 R_{0}$. (In fact, sys $(X) \leq 2 R_{0}$ as the projection map $B_{\tilde{X}}\left(\tilde{x}, R_{0}+\epsilon\right) \rightarrow B_{X}\left(x, R_{0}+\epsilon\right)$ is not injective for some $\tilde{x} \in \tilde{X}$ and $\epsilon$ arbitrary; hence, there exists a curve of length smaller than $2\left(R_{0}+\epsilon\right)$ which projects into a noncontractible closed curve of $X$. The converse inequality is similar.) What is more surprising is that, if $X$ is a normal covering of some fixed, compact, negatively curved manifold $X_{0}$, then knowing the asymptotic deficit $\Omega(X)=\omega(\tilde{X})-\omega(X)$ is enough to deduce an estimate of sys $(X)$ :

Theorem 2 Let $X \rightarrow X_{0}$ be a normal covering of a $n$-dimensional compact manifold $X_{0}$ with $k\left(X_{0}\right) \leq-1, \operatorname{inj}\left(X_{0}\right) \geq r_{0}$ and $\operatorname{vol}\left(X_{0}\right) \leq v_{0}$. Then

$$
\operatorname{sys}(X) \geq C\left(n, r_{0}, v_{0}\right) \log \left[\frac{1}{\Omega(X)}\right]
$$

where $C\left(n, r_{0}, v_{0}\right)$ is a positive function only depending on the base manifold $X_{0}$, via the parameters $n, r_{0}, v_{0}$.

There exist other results relating systole and entropy of a Riemannian manifold (see Katz and Sabourau [7] and Brunnbauer [3]); however, as far as we know, they all concern closed manifolds and give upper bounds of the systole in term of the entropy, whereas here we are interested in estimates from below of the systole for open manifolds. 
Actually, the meaning of formula (1) is the following. Let $X_{0}$ be a fixed compact, negatively curved manifold, and let $X$ be a normal covering of $X_{0}$; then the closer $\omega(X)$ is to the maximal value $\omega(\tilde{X})$, the bigger must the systole of $X$. This can be seen as a property of continuity of the systole in a weak (asymptotic) topology on the set of normal coverings, as (1) implies that $\operatorname{sys}(X) \rightarrow+\infty$ if $\Omega(X) \rightarrow 0$. Notice that, setting $c\left(n, r_{0}, v_{0}\right)=C\left(n, r_{0}, v_{0}\right)^{-1}$, formula (1) is equivalent to the lower bound $\Omega(X) \geq e^{-c\left(n, r_{0}, v_{0}\right) \cdot \operatorname{sys}(X)}$.

Remark 3 A more explicit estimate of $\Omega(X)$, in terms of sys $(X), n, r_{0}, v_{0}$ and of $d_{0}=\operatorname{diam}\left(X_{0}\right)$, is given by formula (17). The function $c\left(n, r_{0}, v_{0}\right)$ is then obtained by showing that $\operatorname{diam}\left(X_{0}\right)$ is bounded by a function $d_{0}\left(n, r_{0}, v_{0}\right)$, and plugging it into (17).

Let now $c$ be some fixed, free homotopy class of closed paths in a compact, negatively curved manifold $X_{0}$. Formula (1) above may be interpreted as a lower bound for the length of the closed geodesic representing $c$ (which is the curve of minimal length in the class), in terms of an asymptotic invariant attached to $c$. Namely, recall that the set of free homotopy classes of closed curves in $X_{0}$ can be identified with the set $C \pi_{1}\left(X_{0}, x_{0}\right)$ of conjugacy classes of $\pi_{1}\left(X_{0}, x_{0}\right)$; therefore, for any choice of $x_{0} \in X_{0}$, the class $c$ naturally determines a normal Riemannian covering $X_{c}$ of $X_{0}$, which is the covering associated to the smallest normal subgroup containing the conjugacy class of $c$. A different choice of the base point $x_{0}$ yields an isometrically isomorphic covering. Then we have the following corollary:

Corollary 4 Let $X_{0}$ be a closed, $n$-dimensional manifold with $k\left(X_{0}\right) \leq-1$ and $\operatorname{inj}\left(X_{0}\right) \geq r_{0}, \operatorname{vol}\left(X_{0}\right) \leq v_{0}$, and let $c$ be any fixed free homotopy class in $C \pi_{1}\left(X_{0}\right)$. Then the minimal closed geodesic $\gamma$ representing $c$ satisfies

$$
\ell(\gamma) \geq C\left(n, r_{0}, v_{0}\right) \log \left[\frac{1}{\Omega\left(X_{c}\right)}\right]
$$

where $X_{c}$ is the normal Riemannian covering associated with $c$.

Actually, if $X_{\gamma} \rightarrow X_{0}$ denotes the covering associated with the infinite cyclic subgroup of $\pi_{1}\left(X_{0}\right)$ generated by $\gamma$, then by covering theory $\ell(\gamma)=\operatorname{sys}\left(X_{\gamma}\right) \geq \operatorname{sys}\left(X_{c}\right)$, and (2) follows from (1). Clearly, this formula is significant only when $\omega\left(X_{c}\right)$ is sufficiently close to $\omega(\tilde{X})$.

We shall now see some consequences of Theorem 1 for normal coverings of negatively curved, locally symmetric spaces. Recall that these are precisely the regular quotients of the hyperbolic space forms $\mathbf{K} \mathbb{H}^{m}$ over the algebras $\mathbf{K}$ of real, complex, quaternionic 
or Cayley numbers (in this last case, $m=2$ ); we shall refer to them as to $\mathbf{K}$-hyperbolic manifolds. It is well known that their curvature can be normalized so that $-4 \leq$ $k\left(\mathbf{K} \mathbb{H}^{m}\right) \leq-1$ and that, if $d=\operatorname{dim}_{\mathbf{R}} \mathbf{K}$, then

$$
\omega\left(\mathbf{K} \mathbb{H}^{m}\right)=m+d-2=\omega_{\mathbf{K}, m} \quad \lambda_{0}\left(\mathbf{K}^{m}\right)=\frac{1}{4} \omega_{\mathbf{K}, m}^{2}
$$

where $\lambda_{0}(X)$ denotes the greatest lower bound of the spectrum of the Laplace-Beltrami operator, that is,

$$
\lambda_{0}(X)=\inf \left\{\frac{\int_{X} f \cdot \Delta f d v}{\int_{X} f^{2} d v} \mid f \in C_{0}^{\infty}(X)\right\} .
$$

For a $\mathbf{K}$-hyperbolic manifold $\mathcal{X}=\Gamma \backslash \mathbf{K} \mathbb{H}^{m}$ there is an elegant formula of Sullivan [11] (for $\mathbf{K}=\mathbf{R}$ ) and Corlette [4] (for $\mathbf{K}=\mathbf{C}, \mathbf{H}$ or $\mathbf{C a}$ ) expressing $\lambda_{0}(\mathcal{X})$ in terms of the exponential growth rate of $\Gamma$ :

$$
\lambda_{0}(\mathcal{X})= \begin{cases}\lambda_{0}\left(\mathbf{K} \mathbb{H}^{m}\right) & \text { if } \omega(\Gamma) \leq \frac{1}{2} \omega_{\mathbf{K}, m} \\ \omega(\Gamma)\left(\omega_{\mathbf{K}, m}-\omega(\Gamma)\right) & \text { if } \omega(\Gamma) \geq \frac{1}{2} \omega_{\mathbf{K}, m}\end{cases}
$$

This implies that the bottom of the spectrum of a quotient of $\mathbf{K} \mathbb{H}^{m}$ by a discrete, torsionless group $\Gamma$ of isometries is the same as for $\mathbf{K} \mathbb{H}^{m}$ if $\Gamma$ has not a sufficiently large exponential growth rate. However, analogously to what we have seen for the exponential growth rate, the bottom of the spectrum always descends when $\mathcal{X}=$ $\Gamma \backslash \mathbf{K} \mathbb{H}^{m}$ is a normal covering of a compact manifold:

Corollary 5 Let $\mathcal{X}=\Gamma \backslash \mathbf{K} \mathbb{H}^{m} \rightarrow \mathcal{X}_{0}$ be a normal, non-simply connected covering of some compact $\mathbf{K}$-hyperbolic manifold $\mathcal{X}_{0}$ with $\operatorname{vol}\left(\mathcal{X}_{0}\right) \leq v_{0}$. Then

(i) $\lambda_{0}(\mathcal{X}) \leq \lambda_{0}\left(\mathbf{K} \mathbb{H}^{m}\right)-\frac{1}{4} e^{-2 c_{\mathbf{K}, m}\left(v_{0}\right) \cdot \operatorname{sys}(\mathcal{X})}$

(ii) $\omega(\Gamma) \geq \frac{1}{2}\left(\omega_{\mathbf{K}, m}+e^{-c_{\mathbf{K}, m}\left(v_{0}\right) \cdot \operatorname{sys}(\mathcal{X})}\right)$

for some positive, explicitable universal functions $c_{\mathbf{K}, m}\left(v_{0}\right)$.

T Roblin [9] has also recently shown, using conformal densities, the strict inequality $\omega(\Gamma)>\frac{1}{2} \omega\left(\Gamma_{0}\right)$ in the more general framework of normal subgroups $\Gamma$ of a discrete, divergent group of isometries $\Gamma_{0}$ of any negatively curved space. However, formula (ii) has the advantage to show and explicitly express the relation between the exponential growth rate of $\Gamma$ and the systole of the quotient space $X$.

Acknowledgments I am grateful to S Gallot for several stimulating discussions, and especially to F Dal'Bo for her constant support and her attentive revision of this paper. 


\section{Basic facts on negatively curved geometry}

We collect here, for the convenience of the reader, some basic results which we shall use in the following chapters. Everywhere in this chapter $\tilde{X}$ is a complete, simply connected Riemannian manifold of sectional curvature $k(\tilde{X}) \leq-1$. We shall denote by $\angle_{x} y, z$ the (unoriented) angle at $x$ between the geodesics $\alpha, \beta$ connecting respectively the point $x$ to $y$ and the point $x$ to $z$.

Proposition 6 Let $A B C$ be a geodesic triangle in $\tilde{X}$. Then

$$
d(A, B) \geq d(A, C)+d(C, B)-d(\vartheta)
$$

where $\vartheta=\angle_{C} A, B$ and $d(\vartheta)=\log (2 /(1-\cos \vartheta))$. In particular, when $\vartheta \geq \pi / 2$ we always have $d(A, B) \geq d(A, C)+d(C, B)-\log 2$.

Proof Let $a=d(A, C)$ and $b=d(C, B)$. By comparing with a geodesic triangle $A^{*} B^{*} C^{*}$ with $d\left(A^{*}, C^{*}\right)=a, d\left(C^{*}, B^{*}\right)=b$ and $\angle_{C^{*}} A^{*}, B^{*}=\vartheta$ in the space form of constant curvature -1 we obtain by Rauch's theorem

$$
\cosh d(A, B) \geq \cosh a \cosh b-\cos \vartheta \sinh a \sinh b
$$

and the equality holds precisely when $k(\tilde{X})=-1$. On the other hand,

$$
\cosh a \cosh b-\cos \vartheta \sinh a \sinh b \geq \cosh (a+b-d(\vartheta)) .
$$

Actually, developing (4) and dividing by $e^{-(a+b)}$, it is enough to verify that

$$
p(T)=\left(2 e^{d(\vartheta)}+\cos \vartheta-1\right) T^{2}-2(1+\cos \vartheta) T+2 e^{-d(\vartheta)}+\cos \vartheta-1 \leq 0
$$

in $T=e^{-(a+b)}$. Notice that $T \geq 0$ and that we may assume $T \leq e^{-d(\vartheta)}$ (otherwise, Proposition 6 is trivially satisfied): therefore, as $p\left(e^{-d(\vartheta)}\right)=(\cos \vartheta-1)\left(e^{-d(\vartheta)}-1\right)^{2} \leq 0$ and $p(0)=0$, it follows that $p(T) \leq 0$.

Proposition 7 Let $A B C$ be a right geodesic triangle in $\tilde{X}$ with angles at vertices $\alpha, \beta$ and $\gamma=\pi / 2$, and opposite sides of lengths $a, b, c$ respectively. Then

$$
\tan \alpha \leq \tanh a / \sinh b .
$$

Proof Consider a comparison triangle $A^{*} B^{*} C^{*}$ in $\mathbf{R} \mathbb{H}^{n}$, that is such that $d\left(B^{*}, C^{*}\right)=$ $a, d\left(A^{*}, C^{*}\right)=b, d\left(A^{*}, B^{*}\right)=c$, and call $\alpha^{*}, \beta^{*}$ and $\gamma^{*}$ the corresponding angles. Then call $P^{*}$ the projection of $C^{*}$ on the geodesic $r$ supporting $A^{*} B^{*}$, and $Q^{*}$ the intersection of $r$ with the geodesic normal to $A^{*} C^{*}$ issuing from $C^{*}$. By Rauch's comparison theorem we deduce $\alpha^{*} \geq \alpha, \beta^{*} \geq \beta$ and $\gamma^{*} \geq \pi / 2$. Therefore, the projection $P^{*}$ falls on the segment $A^{*} B^{*}$, and $Q^{*}$ falls between $P^{*}$ and $B^{*}$. 
Moreover, the distance to a point along a geodesic being a convex function in negative curvature, when a point $R^{*}$ runs from $P^{*}$ and $B^{*}$ the distance $d\left(R^{*}, C^{*}\right)$ grows; hence, $a^{\prime}=d\left(Q^{*}, C^{*}\right) \leq a$. But, then, we get

$$
\tan \alpha \leq \tan \alpha^{*}=\frac{\tanh a^{\prime}}{\sinh b} \leq \frac{\tanh a}{\sinh b}
$$

by standard hyperbolic trigonometry.

If $\tilde{\eta}$ is a geodesic of $\tilde{X}$ and $\tilde{x} \in \tilde{\eta}$, we call geodesic hypersurface orthogonal to $\tilde{\eta}$ at $\tilde{x}$ the smooth hypersurface swept by all geodesics issuing from $\tilde{x}$ orthogonally to $\tilde{\eta}$ (notice that this is not a totally geodesic hypersurface if $k(\tilde{X})$ is not constant).

Proposition 8 Let $\tilde{\eta}$ be a geodesic of $\tilde{X}$ parameterized by arclength, and let $\tilde{x}_{r}=\tilde{\eta}(r)$. Let $H_{r}$ be the geodesic hypersurface orthogonal to $\tilde{\eta}$ at $\tilde{x}_{r}$, and let $U_{r}$ be the connected component of $\tilde{X} \backslash H_{r}$ containing $\widetilde{\eta}(2 r)$. Then:

(i) if $x \in \overline{U_{r}}$ with $r>0$, then $\angle_{\tilde{x}_{0}} x, \tilde{\eta}(+\infty) \leq v(r)$, where $\tan v(r)=1 / \sinh r$;

(ii) if $x \in \overline{U_{r}}$ and $y \in \overline{U_{-r}}$, then $\angle_{\tilde{x}_{0}} x, y \geq \vartheta(r)$, where $\vartheta(r)=\pi-2 v(r)$ and $\cos \vartheta(r)=1-2 \tanh ^{2} r$.

Proof Part (i) immediately follows from Proposition 7, taking $A=\tilde{x}_{0}, C=\tilde{x}_{r}$, and $B$ equal to the intersection between the geodesic segment $\tilde{x}_{0} x$ and $H_{r}$. To prove part (ii), remark that the angle $\angle_{\tilde{x}_{0}} x, y$ satisfies:

$$
\angle \tilde{x}_{0} x, y \geq \angle \tilde{x}_{0} \tilde{\eta}(-\infty), \tilde{\eta}(+\infty)-\angle \tilde{x}_{0} x, \tilde{\eta}(+\infty)-\angle \tilde{x}_{0} y, \tilde{\eta}(-\infty) \geq \pi-2 v(r) .
$$

Hence $\cos \angle_{\tilde{x}_{0}} x, y \leq \cos (\pi-2 v(r))=1-2 /\left(1+\tan ^{2} v(r)\right)=1-2 \tanh ^{2} r$ by (i).

Finally, another classical property we shall use is that, in negative curvature, quasiminimizing curves stay at bounded distance from geodesics:

Proposition 9 (Ghys and de la Harpe [5]) Let $\alpha$ be a geodesic, and let $\beta$ be a curve with same endpoints as $\alpha$ satisfying $\ell(\beta) \leq \ell(\alpha)+\ell$. Then $\beta$ is contained in the $(\ell / 2+\log 3)$-neighborhood of $\alpha$.

\section{Twisted symmetry and twisted product}

Again, throughout all this section $\tilde{X}$ will denote a complete, simply connected Riemannian manifold with $k(\tilde{X}) \leq-1$. 
In what follows, we shall fix once and for all a hyperbolic isometry $\eta$ of $\tilde{X}$ and an origin $\tilde{X} \in \tilde{X}$ on the axis of $\eta$ (that is, the unique geodesic $\tilde{\eta}$ preserved by $\eta$ ). For any point $x \in \tilde{X}$ and any isometry $g \in \operatorname{Is}(\tilde{X})$ we shall denote by $|x|$ and $\|g\|$ respectively the distances $d(\tilde{x}, x)$ and $d(\tilde{x}, g(\tilde{x}))$. Notice that \|\| is not a true norm on $\operatorname{Is}(\tilde{X})$ when $\tilde{x}$ is fixed by some nontrivial isometry; this semi-norm naturally induces a left-invariant pseudometric on $\operatorname{Is}(\tilde{X})$ that we shall still denote by $d$.

Let now $H_{0}$ be the geodesic hypersurface orthogonal to $\tilde{\eta}$ at $\tilde{x}$, and let $H_{i}=\eta^{i} H_{0}$. For $i>0$ define $D_{i}^{*}$ as the open submanifold of $\tilde{X}$ bounded by $H_{i-1}$ and by $H_{i}$, we set $D_{i}=H_{i-1} \cup D_{i}^{*}$, and we call $\tilde{x}_{i}$ the midpoint of the geodesic segment cut by $D_{i}$ on $\tilde{\eta}$. Analogously, for $i<0$ we set $D_{i}^{*}=\eta^{i}\left(D_{1}^{*}\right), D_{i}=H_{i} \cup D_{i}^{*}$ and $\tilde{x}_{i}=\eta^{i}\left(\tilde{x}_{1}\right)$. Notice that each $D_{i}$ is a fundamental domain for the action of the cyclic group generated by $\eta$ on $\tilde{X}$.

We shall write $x>0$ (resp. $x<0$ ) if $x$ belongs to a domain $D_{i}$ with $i>0$ (resp. $i<0)$ and we set, in this case, $\operatorname{sign}(x)=+($ resp. $\operatorname{sign}(x)=-)$. Finally, we shall say that two points $x$ and $y$ are discordant when they belong to different components of $\tilde{X} \backslash D_{i}$, for some $i= \pm 1$.

Definition 10 (Twisted symmetry in $\tilde{X}$ ) We define the twisted symmetry $s_{(\eta, \tilde{x})}: \tilde{X} \rightarrow$ $\tilde{X}$, with respect to $(\eta, \tilde{x})$, as:

$$
s_{(\eta, \tilde{x})}(x)= \begin{cases}\eta^{-2 i-1}(x) & \text { if } x \in D_{i}, i>0 \\ \eta^{-2 i+1}(x) & \text { if } x \in D_{i}, i<0\end{cases}
$$

The points $x$ and $s_{(\eta, \tilde{x})}(x)$ will often be denoted also by $x_{+}$and $x_{-}$respectively, when $(\eta, \tilde{x})$ is clear from the context. Notice that the map $s_{(\eta, \tilde{x})}$ is discontinuous and not involutive; and that we have $\left(D_{i}\right)_{-}=D_{-(i \pm 2)}$ according to $i \gtrless 0$.

Proposition 11 Let $s_{(\eta, \tilde{x})}: \tilde{X} \rightarrow \tilde{X}$ be the twisted symmetry of $\tilde{X}$ with respect to $(\eta, \tilde{x})$, let $L=\|\eta\|$ be the displacement of $\eta$ and $\epsilon(L)=3 L+\log 2$. Then:

(i) $s_{(\eta, \tilde{x})}$ is injective and not surjective;

(ii) $s_{(\eta, \tilde{x})}$ almost preserves the distance to $\tilde{x}$, namely: ||$x|-| x_{-}|| \leq \epsilon(L)$.

Proof If $x_{-}=y_{-}$, then $x, y \in D_{i}$ for some $i$; but the map $D_{i} \rightarrow\left(D_{i}\right)_{-}$is injective (because it coincides with an isometry) hence $x=y$, which proves (i).

To prove (ii), assume for simplicity that $x \in D_{i}, i>0$ (when $x<0$ the proof is analogous). Let $y$ and $z$ be the projections from $x$ and $x_{-}=\eta^{-2 i-1}(x)$ to $\tilde{\eta}$, and let $h=d(x, y)=d\left(x_{-}, z\right)$. Notice that $y$ and $z$ fall, respectively, in the same domains 


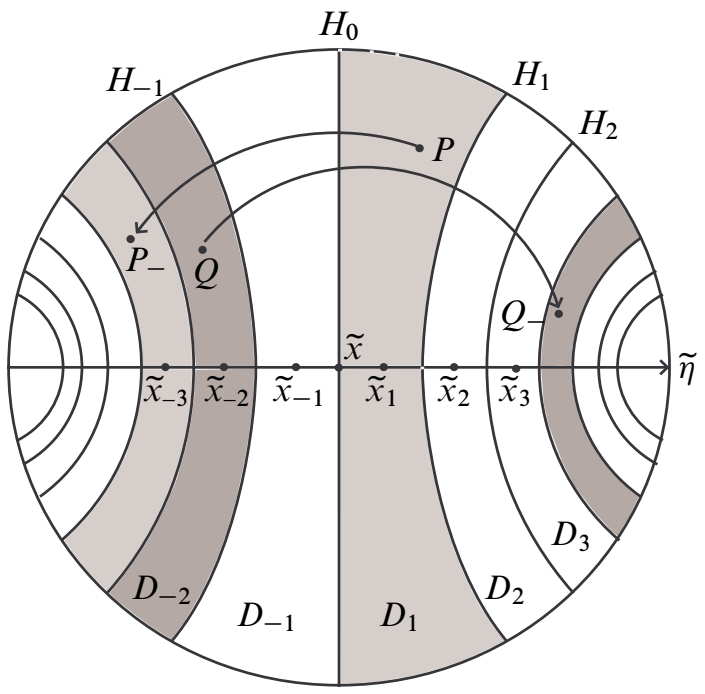

Figure 1: The twisted symmetry of $\tilde{X}$

of $x$ and $x_{-}$, hence $z=\eta^{-2 i-1}(y)=y_{-}$. Actually, assume that, for instance, $y \in D_{j}$ with $j<i$; then, if $p=\overline{x y} \cap H_{i}$ and $q=\tilde{\eta} \cap H_{i}$, the geodesic triangle $y p q$ would have two right angles, which is impossible in negative curvature. So, $d(x, y)=d\left(x_{-}, y_{-}\right)$ (as $\eta$ is an isometry preserving $\tilde{\eta})$. On the other hand, $d(\tilde{x}, y)=d\left(\tilde{x}, \tilde{x}_{i}\right)+\epsilon$ for some $\epsilon \in[-L / 2, L / 2[$, and

$$
d\left(\tilde{x}, y_{-}\right)=d\left(\tilde{x},\left(\tilde{x}_{i}\right)_{-}\right)-\epsilon=d\left(\tilde{x}, \tilde{x}_{i}\right)+2 L-\epsilon .
$$

Thus, by Proposition 6

$$
\begin{aligned}
h+d\left(\tilde{x}, \tilde{x}_{i}\right)+\epsilon-\log 2 & \leq|x| \leq h+d\left(\widetilde{x}, \tilde{x}_{i}\right)+\epsilon \\
h+d\left(\tilde{x}, \tilde{x}_{i}\right)+2 L-\epsilon-\log 2 & \leq\left|x_{-}\right| \leq h+d\left(\tilde{x}, \tilde{x}_{i}\right)-\epsilon+2 L
\end{aligned}
$$

and ||$x_{-}|-| x|| \leq 2|\epsilon|+2 L+\log 2 \leq 3 L+\log 2$.

Proposition 12 Let $s_{(\eta, \tilde{x})}: \tilde{X} \rightarrow \tilde{X}$ be the twisted symmetry of $\tilde{X}$ with respect to $(\eta, \tilde{x}), L=\|\eta\|$ and $\rho(L)=2 \log (\operatorname{coth}(L / 2))+3 L$. For every $x, y \in \tilde{X}$ we have:

(i) either $\{x, y\}$ or $\left\{x, y_{-}\right\}$are separated by a domain $D_{i}$ for some $i \in\{ \pm 1, \pm 2\}$;

(ii) if $\{x, y\}$ are separated by a domain $D_{i}$, for some $i \in\{ \pm 1, \pm 2\}$, then $d(x, y) \geq$ $|x|+|y|-\rho(L)$.

Proof If $x$ and $y$ both lie on the same side of $H_{0}$, then $x, y$ - are separated by $D_{1}$ or $D_{-1}$ by construction. On the other hand, if $x, y$ lie on different sides with respect 
to $H_{0}$, they are separated by $D_{1}$ or $D_{-1}$ unless they belong to $D_{-1} \cup D_{1}$; in this case, $x$ and $y_{-}$are separated by $D_{-2}$ or by $D_{2}$. To prove (ii), notice that, if $D_{i}$ separates $x$ and $y$, then $\angle_{\tilde{x}_{i}} x, y \geq \vartheta(L / 2)$, by Proposition 8 . Therefore,

$$
d(x, y) \geq d\left(x, \tilde{x}_{i}\right)+d\left(\tilde{x}_{i}, y\right)-d(\vartheta(L / 2)) \geq d(x, \tilde{x})+d(\tilde{x}, y)-\rho(L)
$$

for $\rho(L)=3 L+\log (2 /(1-\cos \vartheta(L / 2)))=3 L+2 \log (\operatorname{coth}(L / 2))$.

Definition 13 (Twisted product in $\operatorname{Is}(\tilde{X})$ ) For every $\alpha \in \operatorname{Is}(\tilde{X})$, we set $\alpha_{+}=\alpha$ and define

$$
\alpha_{-}= \begin{cases}\eta^{-2 i-1} \alpha & \text { if } \alpha(\tilde{x}) \in D_{i}, i>0 \\ \eta^{-2 i+1} \alpha & \text { if } \alpha(\tilde{x}) \in D_{i}, i<0 .\end{cases}
$$

Then $\alpha_{-}(\tilde{x})=\alpha(\tilde{x})_{-}$, and we shall say that $\alpha, \beta$ have same or opposite sign according to the sign of $\alpha(\tilde{x}), \beta(\tilde{x})$. If $\alpha, \beta \in \operatorname{Is}(\tilde{X})$ their twisted product is

$$
\alpha * \beta= \begin{cases}\alpha \beta & \text { if } \alpha^{-1}(\tilde{x}), \beta(\tilde{x}) \text { are discordant } \\ \alpha \beta_{-} & \text {otherwise. }\end{cases}
$$

The main properties of,$- *$ on $\operatorname{Is}(\tilde{X})$ are:

(i) the map - almost preserves the norm, ie

$$
\left|\left\|g_{-}\right\|-\|g\|\right| \leq \epsilon(L)
$$

for $\epsilon(L)=3 L+\log 2$ (by Proposition 11);

(ii) for $\alpha * \beta$ the triangular inequality is almost an equality, ie if $\alpha * \beta=\alpha \beta_{\tau}$ with $\tau \in$ $\{ \pm\}$, then

$$
\begin{aligned}
\|\alpha\|+\left\|\beta_{\tau}\right\|-\rho(L) & \leq\|\alpha * \beta\| \leq\|\alpha\|+\left\|\beta_{\tau}\right\| \\
\|\alpha\|+\|\beta\|-\epsilon^{\prime}(L) & \leq\|\alpha * \beta\| \leq\|\alpha\|+\|\beta\|+\epsilon(L)
\end{aligned}
$$

for $\rho(L)=2 \log (\operatorname{coth}(L / 2))+3 L$ and $\epsilon^{\prime}(L)=2 \log (\operatorname{coth}(L / 2))+6 L+\log 2$ (Proposition 12).

Remarks 14 The following properties are easy to verify:

(i) If $\beta(\tilde{x})=\tilde{x}$, then $\beta_{-}=\eta^{-3} \beta$.

(ii) We have $\left(\eta^{k}\right)_{-}= \begin{cases}\eta^{-k-3} & \text { if } k \geq 0 \\ \eta^{-k+1} & \text { if } k<0 .\end{cases}$

(iii) For $k \geq 1$, we have $\eta^{k} * \beta= \begin{cases}\eta^{k} \beta & \text { if } \beta(\tilde{x})>0 \\ \eta^{k} \beta_{-} & \text {if } \beta(\tilde{x})<0 .\end{cases}$ 
(iv) For $k \geq 1$, we have $\left(\eta^{k} * \beta\right)_{-}= \begin{cases}\eta^{-k} \beta_{-}=\eta^{-k} * \beta_{-} & \text {if } \beta(\tilde{x})>0 \\ \eta^{-k-4} \beta=\eta^{-k-4} * \beta & \text { if } \beta(\tilde{x})<0 .\end{cases}$

Also notice that the map $\operatorname{Is}(\tilde{X}) \stackrel{-}{\rightarrow} \operatorname{Is}(\tilde{X})$ is injective but not involutive (as well as $\left.s_{(\eta, \tilde{x})}\right)$, and that $*$ has no unit, and it is neither commutative nor associative. Then we shall write $\alpha * \beta * \gamma$ for $\alpha *(\beta * \gamma)$.

A property slightly weaker than usual left cancellation holds:

Proposition 15 If $\alpha * \beta_{1}=\alpha * \beta_{2}$ and $\beta_{1}, \beta_{2}$ have same sign, then $\beta_{1}=\beta_{2}$.

Proof Let $\alpha * \beta_{i}=\alpha\left(\beta_{i}\right)_{\tau_{i}}$, with $\tau_{i} \in\{ \pm\}$. Assume that $\tau_{1}=+$ and $\tau_{2}=-$; then, from $\alpha \beta_{1}=\alpha\left(\beta_{2}\right)_{-}$we would deduce $\beta_{1}=\left(\beta_{2}\right)_{-}$, hence $\beta_{1}$ and $\beta_{2}$ would have opposite sign, which is a contradiction. Therefore $\tau_{1}=\tau_{2}$ necessarily, so we immediately get $\left(\beta_{1}\right)_{\tau_{1}}=\left(\beta_{2}\right)_{\tau_{2}}$, hence $\beta_{1}=\beta_{2}$.

Let now $G$ be a discrete subgroup of $\operatorname{Is}(\tilde{X})$, still endowed with the seminorm $\|g\|=$ $d(\tilde{x}, g(\tilde{x}))$. If $N$ is a normal subgroup of $G$, the quotient group $G / N$ is endowed with the quotient seminorm, denoted again by \|\| , defined as the infimum of norms of all representatives: $\|g N\|=\inf \left\{\left\|g^{\prime}\right\| \mid g^{\prime} \in g N\right\}$.

We shall say that an element $g \in G$ is minimal modulo a normal subgroup $N$ if $\|g\|=\|g N\|$ (ie $g$ is a representative of minimal length in the class $g N$ ), and that $g$ is $\epsilon$-minimal modulo $N$ if $\|g\| \leq\|g N\|+\epsilon$. We shall also denote by $\mathcal{N}_{G}(g)$ the normal subgroup of $G$ generated by an element $g$.

Remark 16 Notice that if $\eta \in G$, then the twisted symmetry $s_{(\eta, \tilde{x})}$ of $\tilde{X}$ induces the identity map on the quotient $G \backslash \tilde{X} \rightarrow G \backslash \tilde{X}$ (as $(G x)_{-}=G x$ ). Moreover, if $\eta \in G$, notice that the map $G \stackrel{-}{\rightarrow} G$ associated with $s_{(\eta, \widetilde{x})}$ induces the identity on the quotient $G / \mathcal{N}_{G}(\eta)$, ie $g \mathcal{N}_{G}(\eta)=g_{-} \mathcal{N}_{G}(\eta)$ for all $g \in G$.

We conclude this section with the following proposition, which is crucial for the proof of Theorem 1. As explained in the introduction, the proof will consist in injecting the space $\ell_{c}^{1}(G / N)$ of finite, arbitrarily long sequences of elements of $G / N$ (see Section 4 for a precise definition) in $G$ by a contracting map $j$. This will be achieved by lifting any sequence of elements $\left(\gamma_{i}\right)$ of $G / N$ to minimal representatives in $G$ and then enchaining them, interposing between them suitable powers $\eta_{i}^{k}$ of some $\eta \in N$. A repeated application of the proposition below will enable us to recover the whole sequence of $\gamma_{i}$ 's from the knowledge of $j\left(\gamma_{1}, \ldots, \gamma_{m}\right)$, thus showing the injectivity of the map $j$. Formally, the proposition says that if the equation $\alpha_{1} * \eta^{k} * \beta_{1}=\alpha_{2} * \eta^{k} * \beta_{2}$ holds in the discrete group $G \subset \operatorname{Is}(\tilde{X})$ (for $k$ large enough and the $\alpha_{i}$ minimal modulo $\left.\mathcal{N}_{G}(\eta)\right)$, then we can almost recover the identity $\alpha_{1}=\alpha_{2}$. 
Proposition 17 Let $\kappa$ be the smallest integer $\kappa \geq \kappa(L)=(7 / L) \log \left(3 e^{2 L} \operatorname{coth}(L / 2)\right)$, and set $\delta(L)=9 \log \left(3 e^{3 L} \operatorname{coth}(L / 2)\right)$. Let $G$ be a discrete group of $\operatorname{Is}(\tilde{X})$, let $\alpha_{i}, \beta_{i}$ and $\eta \in G$, and assume that the $\alpha_{i}$ 's are $\epsilon(L)$-minimal modulo $\mathcal{N}_{G}(\eta)$. If $\alpha_{1} * \eta^{\kappa} * \beta_{1}=\alpha_{2} * \eta^{\kappa} * \beta_{2}$ then $d\left(\alpha_{1}, \alpha_{2}\right)<\delta(L)$.

The informal idea behind the proof of Proposition 17 is to look, on the geodesic of $\tilde{X}$ representing $g=\alpha_{i} * \eta^{k} * \beta_{i}$, for the first point $\tilde{z}$ such that the geodesic $\tilde{x} \widetilde{z}$ no longer minimizes when projected on the quotient space $X=\tilde{X} / \mathcal{N}_{G}(\eta)$. Then as the $\alpha_{i}$ 's are minimal modulo $\mathcal{N}_{G}(\eta)$, we shall be able to find the points $\alpha_{i}(\tilde{x})$ at finite distance (depending on $L=\|\eta\|$ ) from $\widetilde{z}$, and therefore the $\alpha_{i}$ will be almost determined.

Proof of Proposition 17 Let $\gamma=\alpha_{i} * \eta^{\kappa} * \beta_{i}=\alpha_{i}\left(\eta^{\kappa} * \beta_{i}\right)_{\sigma_{i}}$ for $\sigma_{i} \in\{ \pm\}$; by Remarks 14 (iii)-(iv) we have $\left(\eta^{\kappa} * \beta_{i}\right)_{\sigma_{i}}=\eta^{\kappa_{i}}\left(\beta_{i}\right)_{\tau_{i}}=\eta^{\kappa_{i}} *\left(\beta_{i}\right)_{\tau_{i}}$, for some $\kappa_{i} \in\{ \pm \kappa,-\kappa-4\}$ and $\tau_{i} \in\{ \pm\}$. Let now $\tilde{\alpha}_{i}, \tilde{\eta}_{i}, \widetilde{\beta}_{i}$ be respectively the geodesic segments from $\tilde{x}$ to $A_{i}=\alpha_{i}(\tilde{x})$, from $A_{i}$ to $B_{i}=\alpha_{i}\left(\eta^{\kappa_{i}}(\tilde{x})\right)$, and from $B_{i}$ to $\gamma(\tilde{x})$. Let then $\tilde{\gamma}$ and $\tilde{\gamma}_{i}$ be the geodesic segments respectively from $\tilde{x}$ and from $A_{i}$ to $\gamma(\tilde{x})$. Finally, let $x_{i}$ and $y_{i}$ be the projections of $A_{i}$, and $B_{i}$ on $\tilde{\gamma}$. See Figure 2.

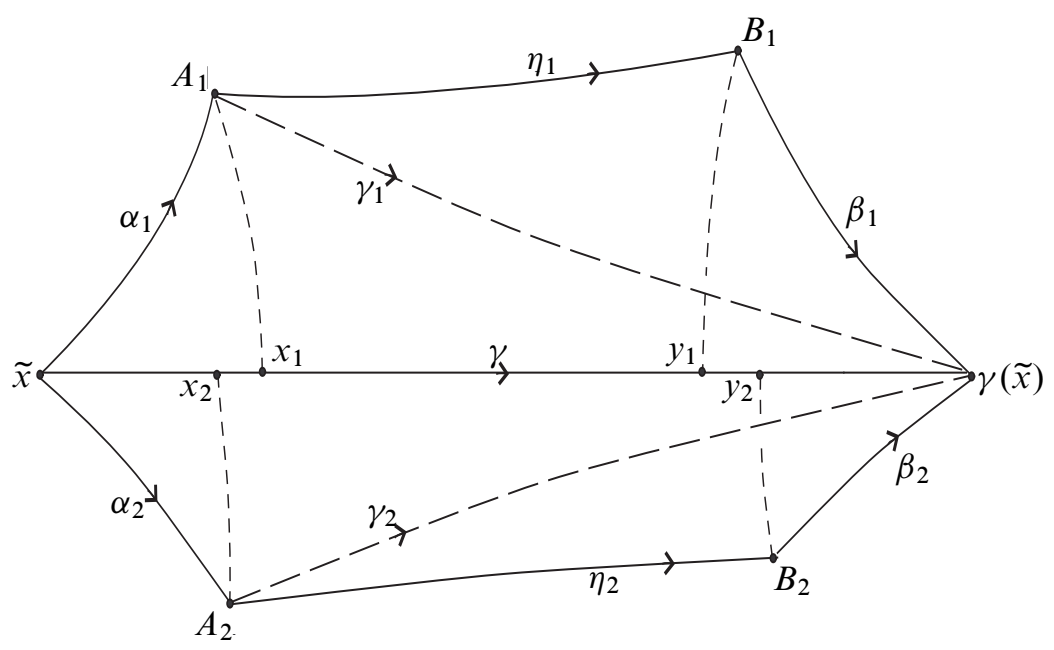

Figure 2

We have, by (7)

$$
\ell\left(\tilde{\alpha}_{i}\right)+\ell\left(\tilde{\gamma}_{i}\right)=\left\|\alpha_{i}\right\|+\left\|\eta^{\kappa_{i}}\left(\beta_{i}\right)_{\tau_{i}}\right\| \leq\left\|\alpha_{i} *\left(\eta^{\kappa} * \beta_{i}\right)\right\|+\rho(L)=\ell(\tilde{\gamma})+\rho(L)
$$

as well as, by Remarks 14 (iv),

$$
\ell\left(\widetilde{\alpha}_{i}\right)+\ell\left(\widetilde{\eta}_{i}\right)+\ell\left(\widetilde{\beta}_{i}\right) \leq\left\|\alpha_{i}\right\|+\left\|\left(\eta^{\kappa} * \beta_{i}\right)_{\sigma_{i}}\right\|+\rho(L) \leq\left\|\alpha_{i} *\left(\eta^{\kappa} * \beta_{i}\right)\right\|+2 \rho(L) .
$$


Hence, by Proposition 9

while

$$
\begin{aligned}
& d\left(A_{i}, x_{i}\right)=d\left(A_{i}, \tilde{\gamma}\right) \leq \rho(L) / 2+\log 3 \\
& d\left(B_{i}, y_{i}\right)=d\left(B_{i}, \tilde{\gamma}\right) \leq \rho(L)+\log 3 \\
& d\left(A_{i}, B_{i}\right)=\left\|\eta^{\kappa_{i}}\right\| \in[\kappa L,(\kappa+4) L] .
\end{aligned}
$$

Since projections on convex subsets reduce the distance in negative curvature, the previous equations then yield

$$
\kappa L-3 \rho(L) / 2-2 \log 3 \leq d\left(x_{i}, y_{i}\right) \leq d\left(A_{i}, B_{i}\right) \leq(\kappa+4) L .
$$

Consider now the projection $\pi: \tilde{X} \rightarrow X=\mathcal{N}_{G}(\eta) \backslash \tilde{X}$ (with the quotient distance). The action of $G$ on $\tilde{X}$ induces an action of $G / \mathcal{N}_{G}(\eta)$ on $X$, and we clearly have $\pi\left(A_{i}\right)=\pi\left(B_{i}\right)$ (as $\mathcal{N}_{G}(\eta)$ is normal). Therefore, by (9) and (10) again we deduce

$$
d\left(\pi\left(x_{i}\right), \pi\left(y_{i}\right)\right) \leq 3 \rho(L) / 2+2 \log 3 .
$$

The inequalities (11) and (12), in turn, yield

$$
d\left(x_{i}, y_{i}\right)-d\left(\pi\left(x_{i}\right), \pi\left(y_{i}\right)\right) \geq \kappa L-3 \rho(L)-4 \log 3 .
$$

Now, remark that each $y_{i}$ follows $x_{i}$ along $\tilde{\gamma}$; otherwise, by (10), we would have

$$
\begin{aligned}
\left\|\left(\beta_{i}\right)_{\tau}\right\|=d\left(B_{i}, \gamma(\tilde{x})\right) & \geq d\left(y_{i}, \gamma(\tilde{x})\right) \geq d\left(x_{i}, \gamma(\tilde{x})\right) \\
& \geq d\left(A_{i}, \gamma(\tilde{x})\right)-(\rho(L) / 2+\log 3) \\
& =\left\|\left(\eta^{\kappa} * \beta_{i}\right)_{\sigma_{i}}\right\|-(\rho(L) / 2+\log 3),
\end{aligned}
$$

but, on the other hand, by the main property of $*$,

$$
\left\|\left(\eta^{\kappa} * \beta_{i}\right)_{\sigma_{i}}\right\|=\left\|\eta^{\kappa_{i}} *\left(\beta_{i}\right)_{\tau_{i}}\right\| \geq\left\|\eta^{\kappa_{i}}\right\|+\left\|\left(\beta_{i}\right)_{\tau_{i}}\right\|-\rho(L) \geq \kappa L+\left\|\left(\beta_{i}\right)_{\tau_{i}}\right\|-\rho(L) .
$$

Hence $\kappa \leq(3 \rho(L) / 2+\log 3) / L$, which contradicts our choice of $\kappa$.

Moreover, the couple $\left(y_{1}, y_{2}\right)$ follows the couple of points $\left(x_{1}, x_{2}\right)$ along $\tilde{\gamma}$. Actually, assume for instance that $x_{2}$ is between $y_{1}$ and $y_{2}$; then, by the $\epsilon(L)$-minimality modulo $\mathcal{N}_{G}(\eta)$ of $\alpha_{2}$ and by (13)

$$
\begin{aligned}
& \left\|\alpha_{2} \mathcal{N}_{G}(\eta)\right\|+\epsilon(L) \\
& \quad \geq\left\|\alpha_{2}\right\|=d\left(\tilde{x}, A_{2}\right) \geq d\left(\tilde{x}, x_{2}\right)=d\left(\tilde{x}, x_{1}\right)+d\left(x_{1}, y_{1}\right)+d\left(y_{1}, x_{2}\right) \\
& \quad \geq d\left(\pi(\tilde{x}), \pi\left(x_{1}\right)\right)+d\left(\pi\left(x_{1}\right), \pi\left(y_{1}\right)\right)+\kappa L-3 \rho(L)-4 \log 3+d\left(\pi\left(y_{1}\right), \pi\left(x_{2}\right)\right) \\
& \quad \geq d\left(\pi(\tilde{x}), \pi\left(A_{2}\right)\right)+\kappa L-7 \rho(L) / 2-5 \log 3 \\
& \quad \geq\left\|\alpha_{2} \mathcal{N}_{G}(\eta)\right\|+\kappa L-7 \rho(L) / 2-5 \log 3
\end{aligned}
$$

hence $\kappa \leq(7 \rho(L) / 2+\epsilon(L)+5 \log 3) / L$, which again contradicts the choice of $\kappa$. 
Concluding, we deduce that $d\left(x_{1}, x_{2}\right) \leq d\left(x_{1}, y_{1}\right) \leq(\kappa+4) L$, by (11); so, by (9),

$$
d\left(\alpha_{1}, \alpha_{2}\right) \leq d\left(A_{1}, x_{1}\right)+d\left(x_{1}, x_{2}\right)+d\left(x_{2}, A_{2}\right) \leq \rho(L)+2 \log 3+(\kappa+4) L<\delta(L) .
$$

\section{Growth of nets in normal coverings}

Recall that a subset $S$ of a metric space $(X, d)$ is called a $\delta$-net if, for all $x \in X$, we have $d(x, S) \leq \delta$. For instance, if a discrete group $\Gamma$ acts on $X$ with compact quotient $X_{0}=\Gamma \backslash X$, and $\operatorname{diam}\left(X_{0}\right) \leq \delta$, any orbit of $\Gamma$ is a $\delta$-net in $X$. On the other hand, a subset $S$ of $(X, d)$ is called a $\delta$-separated set if all the open balls $B_{S}(s, \delta)$ of radius $\delta$ centered at points of $S$ are disjoint (hence, $d\left(s, s^{\prime}\right) \geq 2 \delta$ for all $s, s^{\prime} \in S$ ). Notice that any maximal $\delta$-separated set $S$ of $(X, d)$ is a $2 \delta$-net, by maximality.

In this section we shall be interested in estimates on the growth of nets in normal coverings. We shall use the following notation: for a general, discrete metric space $S$ whose balls of finite radius are finite, the growth function of $S$ and the exponential growth rate of $S$ are defined, respectively, as

$$
v_{S}(s, R)=\operatorname{card} B_{S}(s, R), \quad \omega(S)=\limsup _{R \rightarrow+\infty} R^{-1} \cdot \log v_{S}(s, R)
$$

where $B_{S}(s, R)$ denotes, as usual, the subset of elements whose distance from $s$ is smaller than $R$. Moreover, for a Riemannian manifold $X$ and any discrete subset $S \subset X$ we define, for all $\sigma \geq 0$ :

$$
\begin{array}{llrl}
A_{X}^{\sigma}(x, R) & =\overline{B_{X}(x, R+\sigma)} \backslash \overline{B_{X}(x, R-\sigma)}, & & v_{X}^{\sigma}(x, R)=\operatorname{vol} A_{X}^{\sigma}(x, R) \\
A_{S}^{\sigma}(x, R) & =A_{X}^{\sigma}(x, R) \cap S, & v_{S}^{\sigma}(x, R) & =\operatorname{card} A_{S}^{\sigma}(x, R)
\end{array}
$$

Remark 18 It is classical that, for a normal Riemannian covering $X$ of a closed Riemannian manifold $X_{0}$ we have:

$$
\omega(X)=\lim _{R \rightarrow \infty} R^{-1} \cdot \log v_{X}(x, R)=\lim _{R \rightarrow \infty} R^{-1} \cdot \log v_{X}^{\sigma}(x, R)
$$

(the existence of the second limit is justified by Proposition 20 below). Actually, we have

$$
B_{X}(x, 2 N \sigma)=\bigsqcup_{n=0}^{N} A_{X}^{\sigma}(x, 2 n \sigma)
$$

so, $v_{X}(x, 2 N \sigma)=\sum_{n} v_{X}^{\sigma}(x, 2 n \sigma)$. But now, if $\omega=\lim _{R \rightarrow \infty} R^{-1} \cdot \log v_{X}^{\sigma}(x, R)$, we know that for all $\epsilon>0$ there exist constants $a, b>0$ such that

$$
a e^{(\omega-\epsilon) R} \leq v_{X}^{\sigma}(x, R) \leq b e^{(\omega+\epsilon) R} .
$$


It follows that

$$
e^{2 N \sigma(\omega-\epsilon)} \sim a \sum_{n=0}^{N} e^{2 n \sigma(\omega-\epsilon)} \leq v_{X}(x, 2 N \sigma) \leq b \sum_{n=0}^{N} e^{2 n \sigma(\omega+\epsilon)} \sim e^{2 N \sigma(\omega+\epsilon)}
$$

and, as $\epsilon$ is arbitrary, passing to limits yields $\omega=\lim _{R \rightarrow \infty} R^{-1} \cdot \log v_{X}(x, R)$. Similarly, if $S$ is a discrete subset of $X$, then we also have

$$
\omega(S)=\lim _{R \rightarrow \infty} R^{-1} \cdot \log v_{S}(x, R)=\lim _{R \rightarrow \infty} R^{-1} \cdot \log v_{S}^{\sigma}(x, R)
$$

provided that the second limit exists.

We shall make use of the following estimates, the first of which (à la Bishop-Gromov) is due to G Reviron:

Proposition 19 [8] Let $X$ be a complete Riemannian manifold admitting some properly discontinuous cocompact group of isometries. Then for all $R \geq 2 r>0$ we have:

$$
\frac{\sup _{x \in X} v_{X}(x, R)}{\inf _{x \in X} v_{X}(x, r)} \leq\left[\frac{\sup _{x \in X} v_{X}(x, 4 r)}{\inf _{x \in X} v_{X}(x, r)}\right]^{\frac{R}{2 r}}
$$

Proposition 20 Let $X$ be a complete Riemannian manifold admitting some properly discontinuous cocompact group of isometries $\Gamma$. Let $X_{0}=\Gamma \backslash X$ and $\operatorname{inj}\left(X_{0}\right) \geq r_{0}$, $\operatorname{diam}\left(X_{0}\right) \leq d_{0}$ and $\operatorname{vol}\left(X_{0}\right) \leq v_{0}$. Then for any orbit $\Gamma x$ of $\Gamma$ in $X$ and any $\delta$-net $S$ of $\Gamma x$, we have

(i) $\omega(S)=\omega(\Gamma x)=\omega(X)=\omega$;

(ii) $v_{S}^{\sigma+\delta}(x, R) \geq v_{\Gamma x}^{\sigma}(x, R) / v_{\Gamma x}(x, \delta)$, for all $\sigma, R \geq 0$;

(iii) $v_{\Gamma x}^{2 d_{0}}(x, R) \geq e^{\omega(X) R}$ for all $R \geq 0$.

Moreover, if $k(X) \leq-1$ and $v_{n}(r)$ denotes the volume of balls of radius $r$ in $\mathbb{H}^{n}$, then

$$
\omega \leq \frac{2}{r_{0}} \log \left[\frac{v_{0}}{v_{n}\left(r_{0} / 4\right)}\right] \text { and } v_{S}^{2 d_{0}+\delta}(x, R) \geq\left[\frac{v_{n}\left(r_{0} / 4\right)}{v_{0}}\right]^{\frac{2\left(d_{0}+\delta\right)}{r_{0}}-1} e^{\omega R} .
$$

Proof of Proposition 20 Let $\Omega(y)=\{z \in X \mid d(z, y)<d(z, \gamma(y))\}$ be the open Dirichlet domain, centered at $y \in X$, for the action of $\Gamma$. Then

$$
\bigcup_{y \in A_{\Gamma x}^{\sigma-d_{0}}(x, R)} \Omega(y) \subset A_{X}^{\sigma}(x, R) \subset \bigsqcup_{y \in A_{\Gamma x}^{\sigma+d_{0}}(x, R)} \overline{\Omega(y)}
$$


hence $v_{\Gamma x}^{\sigma-d_{0}}(x, R) \cdot v_{0} \leq v_{X}^{\sigma}(x, R) \leq v_{\Gamma x}^{\sigma+d_{0}}(x, R) \cdot v_{0}$. Moreover, notice that

$$
A_{\Gamma x}^{\sigma}(x, R) \subset \bigcup_{s \in A_{S}^{\sigma+\delta}(x, R)} B_{\Gamma x}(s, \delta),
$$

and that $v_{\Gamma x}(s, \delta)=v_{\Gamma x}(x, \delta)$ for all $s \in S$. Hence, $v_{S}^{\sigma}(x, R) \leq v_{\Gamma x}^{\sigma}(x, R) \leq$ $v_{S}^{\sigma+\delta}(x, R) \cdot v_{\Gamma x}(x, \delta)$. This proves (ii) and, by taking limits, (i).

To show (iii), remark that for all $R_{1}, R_{2}>0$ we have

$$
A_{\Gamma x}^{2 d_{0}}\left(x, R_{1}+R_{2}\right) \subset \bigcup_{y \in A_{\Gamma x}^{2 d_{0}}\left(x, R_{1}\right)} A_{\Gamma x}^{2 d_{0}}\left(y, R_{2}\right) .
$$

Actually, let $z \in A_{\Gamma x}^{2 d_{0}}\left(x, R_{1}+R_{2}\right)$; then, $d(x, z)=R_{1}+R_{2}+\epsilon$, for some $\epsilon \epsilon$ ] $-2 d_{0}, 2 d_{0}$ ]. Let now $x^{\prime}$ be a point on a minimizing geodesic from $x$ to $z$ such that $d\left(x, x^{\prime}\right)=R_{1}+\epsilon / 2$, and let $y \in \Gamma x$ such that $d(x, y) \leq d_{0}$. Then $y \in A_{\Gamma x}^{2 d_{0}}\left(x, R_{1}\right)$ and $z \in A_{\Gamma x}^{2 d_{0}}\left(y, R_{2}\right)$ (because $\left.d\left(x^{\prime}, z\right)=R_{2}+\epsilon / 2\right)$. Since for all $y \in \Gamma x$, we have $\operatorname{card} A_{\Gamma x}^{2 d_{0}}\left(y, R_{2}\right)=\operatorname{card} A_{\Gamma x}^{2 d_{0}}\left(x, R_{2}\right)$, we deduce that

$$
v_{\Gamma x}^{2 d_{0}}\left(x, R_{1}+R_{2}\right) \leq v_{\Gamma x}^{2 d_{0}}\left(x, R_{1}\right) \cdot v_{\Gamma x}^{2 d_{0}}\left(x, R_{2}\right),
$$

that is, $\log v_{\Gamma x}^{2 d_{0}}(x, R)$ is a sub-additive function; this implies that the limit $\omega(\Gamma x)=$ $\lim _{R \rightarrow \infty} R^{-1} \cdot \log v_{\Gamma x}^{2 d_{0}}(x, R)$ exists and it is equal, by (ii), to $\omega(X)$. Best, by subadditivity we deduce

$$
\frac{n \log v_{\Gamma x}^{2 d_{0}}(x, R)}{n R} \geq \frac{\log v_{\Gamma x}^{2 d_{0}}(x, n R)}{n R}
$$

and, taking limits for $n \rightarrow \infty$, we obtain $R^{-1} \log v_{\Gamma x}^{2 d_{0}}(x, R) \geq \omega(\Gamma x)$ for all $R>0$, which proves (iii).

Finally, notice that as $B_{X}\left(x, d_{0}+\delta\right) \supset \bigsqcup_{y \in B_{\Gamma x}(x, \delta)} \Omega(y)$, we have that $v_{\Gamma x}(x, \delta) \leq$ $v_{X}\left(x, d_{0}+\delta\right) / v_{0}$; hence from (ii) and (iii) we deduce:

$$
v_{S}^{2 d_{0}+\delta}(x, R) \geq \frac{v_{0}}{v_{X}\left(x, d_{0}+\delta\right)} e^{\omega R}
$$

Now assume that $k(X) \leq-1$. Using Proposition 19 for $r=r_{0} / 4$ we obtain the estimate $v_{X}(x, R) \leq v_{0} \cdot\left[v_{0} / v_{n}\left(r_{0} / 4\right)\right]^{2 R / r_{0}-1}$ by Bishop-Gunther's comparison theorem. This estimate shows that $\omega \leq\left(2 / r_{0}\right) \log \left[v_{0} / v_{n}\left(r_{0} / 4\right)\right]$, while plugging it into (15) ends the proof.

We shall now prove a general result on the growth of discrete metric spaces whose annuli grow by an exponential law. Let $\left(S, s_{0}\right)$ be any discrete, pointed metric space whose balls of finite radius are finite, and let $S^{*}=S \backslash\left\{s_{0}\right\}$. We consider the space 
$\ell^{1}\left(S^{*}\right)$ of finite, arbitrarily long sequences $\left(s_{k}\right)$ of points of $S^{*}$. Then we define $\ell_{c}^{1}\left(S^{*}\right)$ to be the same space as above, endowed with the "norm"

$$
\left\|\left(s_{1}, \ldots, s_{m}\right)\right\|_{c}=\sum_{k=1}^{m} d\left(s_{k}, s_{0}\right)+(m-1) c .
$$

We can define the "balls of $\ell_{c}^{1}\left(S^{*}\right)$ centered at the empty sequence" as

$$
B_{c}(R)=\left\{\left(s_{k}\right) \mid\left\|\left(s_{k}\right)\right\|_{c} \leq R\right\} .
$$

As $S$ is discrete, these are finite sets for all $R$, so we can set $v_{c}(R)=\operatorname{card} B_{c}(R)$ and consider the exponential growth rate of this space:

$$
\omega\left(\ell_{c}^{1}\left(S^{*}\right)\right)=\limsup _{R \rightarrow \infty} R^{-1} \log v_{c}(R) .
$$

Proposition 21 Let $\left(S, s_{0}\right)$ be a pointed, discrete metric space verifying:

(i) all the balls of finite radius of $S$ are finite sets;

(ii) there exist $\sigma, \lambda, \omega>0$ such that $v_{S}^{\sigma}\left(s_{0}, R\right) \geq \lambda \cdot e^{\omega R}$ for all $R \geq 0$.

Then for every $c>0$ we have

$$
\omega\left(\ell_{c}^{1}\left(S^{*}\right)\right) \geq \omega+\frac{\log \left(1+\lambda e^{-\omega(\sigma+c)}\right)}{2(\sigma+c)} .
$$

Proof Let $B_{c}^{m}(R)$ be the subset of $B_{c}(R)$ of sequences of length $m$, and denote the subset of $B_{c}^{m}(R)$ made up of sequences $\left(s_{k}\right)$ such that $s_{k} \in A_{S^{*}}^{\sigma}\left(s_{0}, R_{k}\right)$ for all $k$ by $A_{S^{*}}^{\sigma}\left(s_{0}, R_{1}\right) \times \cdots \times A_{S^{*}}^{\sigma}\left(s_{0}, R_{m}\right)$. Finally, let $\Delta=\sigma+c$, and for every $k_{i} \in \mathbf{N}^{*}$ set $R_{i}=\left(2 k_{i}-1\right) \Delta$. Then we have a decomposition:

$$
B_{c}(2 M \Delta) \supset \bigsqcup_{m \geq 1}^{M} B_{c}^{m}(2 M \Delta) \supset \bigsqcup_{m \geq 1}^{M} \bigsqcup_{k_{1}, \ldots, k_{m} \geq 1}^{\Sigma_{i} k_{i}=M} A_{S^{*}}^{\sigma}\left(s_{0}, R_{1}\right) \times \cdots \times A_{S^{*}}^{\sigma}\left(s_{0}, R_{m}\right)
$$

Actually, if $\left(s_{k}\right) \in A_{S^{*}}^{\sigma}\left(s_{0}, R_{1}\right) \times \cdots \times A_{S^{*}}^{\sigma}\left(s_{0}, R_{m}\right)$, then we have that $\left\|\left(s_{k}\right)\right\|_{c} \leq$ $\sum_{k}\left[\left(2 k_{i}-1\right) \Delta+\sigma\right]+(m-1) c \leq 2 M \Delta$. Moreover, remark that these are disjoint unions, since $A_{S^{*}}^{\sigma}\left(s_{0}, R_{i}\right) \cap A_{S^{*}}^{\sigma}\left(s_{0}, R_{j}\right)=\varnothing$ if $i \neq j$. Also notice that, as each 
$R_{i} \geq \Delta>\sigma$, we have $A_{S^{*}}^{\sigma}\left(s_{0}, R_{i}\right)=A_{S}^{\sigma}\left(s_{0}, R_{i}\right)$. Therefore, by (ii), we get:

$$
\begin{aligned}
v_{c}(2 M \Delta) & \geq \sum_{m=1}^{M} \sum_{k_{1}, \ldots, k_{m} \geq 1}^{\Sigma_{i} k_{i}=M} v_{S}^{\sigma}\left(s_{0}, R_{1}\right) \cdots v_{S}^{\sigma}\left(s_{0}, R_{m}\right) \\
& \geq \sum_{m=1}^{M} \sum_{\Sigma_{1}, \ldots, k_{m} \geq 1}^{\Sigma_{i} k_{i}=M} \lambda^{m} e^{2 M \Delta \omega} \cdot e^{-m \Delta \omega} \\
& =e^{2 M \Delta \omega} \sum_{m=1}^{M}\left(\begin{array}{l}
M-1 \\
m-1
\end{array}\right) \cdot\left(\lambda e^{-\Delta \omega}\right)^{m} \\
& =\lambda e^{-\Delta \omega} \cdot e^{2 M \Delta \omega} \cdot\left(1+\lambda e^{-\Delta \omega}\right)^{M-1}
\end{aligned}
$$

and thus $\quad \omega\left(\ell_{c}^{1}\left(S^{*}\right)\right) \geq \lim _{M \rightarrow \infty} \frac{\log v_{c}(2 M \Delta)}{2 M \Delta}=\omega+\frac{\log \left(1+\lambda e^{-\Delta \omega}\right)}{2 \Delta}$.

\section{Proof of the main results}

We start with the sequence of normal coverings $\tilde{X} \rightarrow X \rightarrow X_{0}$ of a closed, negatively curved manifold $X_{0}$, and we want to show that $\omega(\tilde{X})>\omega(X)$. If we fix a base point $\tilde{x}$ in $\tilde{X}$, and $x, x_{0}$ are its projections to $X, X_{0}$, the groups of deck transformations $\operatorname{Aut}\left(\tilde{X} \rightarrow X_{0}\right)$ and $\operatorname{Aut}\left(X \rightarrow X_{0}\right)$ can be canonically identified, respectively, with $G=$ $\pi_{1}\left(X_{0}, x_{0}\right)$ and with the quotient $\Gamma=G / N$ by the normal subgroup $N=\pi_{1}(X, x)$ of $G$. We shall systematically identify elements of $G$ and $\Gamma$ with the automorphisms of $\tilde{X}$ and $X$ they represent.

As explained before Remark 16, $G$ and $\Gamma$ carry a natural left-invariant norm (and, consequently, a left-invariant distance), by identifying them with their orbits in the Riemannian manifolds $\tilde{X}$ and $X$; these are true norms, as the deck transformations have no fixed points. With respect to these metric structures we have, by Proposition 20; $\omega(G)=\omega(G \tilde{x})=\omega(\tilde{X})$ and $\omega(\Gamma)=\omega(\Gamma x)=\omega(X)$.

Proofs of Theorem 1 and Theorem 2 Let $\eta$ be a closed geodesic realizing the systole of $X$, ie sys $(X)=\ell(\eta)=L$, and let $\eta_{0}$ be its projection on $X_{0}$ (such geodesics exist by an Ascoli-Arzelà argument, as $X_{0}$ is compact). We chose the base point $x$ of $X$ on $\eta$ and call $\tilde{x}, x_{0}$ respectively a lift of $x$ to $\tilde{X}$ and its projection to $X_{0}$; for this choice of the base point, we clearly have $\|\eta\|=\ell(\eta)$.

Let now $S$ be a maximal $(\delta / 2)$-separated set of the orbit $\Gamma x$ containing $x$, for $\delta=\delta(L)=9 \log \left(3 e^{3 L} \operatorname{coth}(L / 2)\right)$ as in Proposition 17 (such a subset always exists 
by Zorn's Lemma), and set $S^{*}=S \backslash\{x\}$. Since $S$ is maximal, it is a $\delta$-net in $\Gamma$. We shall prove that

Proposition 22 There exists an injection $j: \ell_{c}^{1}\left(S^{*}\right) \rightarrow G$ which contracts the norms, for $c \geq 7 \log \left(5 e^{4 L} \operatorname{coth}(L / 2)\right)$.

Assuming for a moment Proposition 22, we infer that the $R$-balls of $\ell_{c}^{1}\left(S^{*}\right)$ can be injected in the $R$-balls of $G$ (centered at the identity element); therefore

$$
\omega=\omega(\tilde{X})=\omega(G) \geq \omega\left(\ell_{c}^{1}\left(S^{*}\right)\right) .
$$

Now, let $d_{0}=\operatorname{diam}\left(X_{0}\right)$; we know by Proposition 20 that $v_{S}^{\sigma}(x, R) \geq \lambda e^{\omega(X) R}$, for $\sigma=2 d_{0}+\delta$ and $\lambda=\left[v_{n}\left(r_{0} / 4\right) / v_{0}\right]^{2\left(d_{0}+\delta\right) / r_{0}-1}$; hence, Proposition 21 yields

$$
\omega(\tilde{X}) \geq \omega\left(\ell_{c}^{1}(S, x)\right) \geq \omega(X)+\frac{\log \left(1+\lambda e^{-\omega(\sigma+c)}\right)}{2(\sigma+c)}
$$

which proves Theorem 1 .

More precisely, plugging in (16) the value of $\sigma, \lambda$ corresponding to our $\delta=\delta(L)$, the estimate $\omega \leq\left(2 / r_{0}\right) \log \left[v_{0} / v_{n}\left(r_{0} / 4\right)\right]$ of Proposition 20 and $c=7 \log \left(5 e^{4 L} \operatorname{coth}(L / 2)\right)$, we obtain

$$
\Omega(X) \geq \frac{\log \left(1+\left[\frac{v_{n}\left(r_{0} / 4\right)}{v_{0}}\right]^{\left(182+\left(3 d_{0}+33\right) / r_{0}+13 / r_{0}^{2}\right)\left(\operatorname{sys}(X) / r_{0}\right)}\right)}{\left(182+\left(3 d_{0}+33\right) / r_{0}+13 / r_{0}^{2}\right) \operatorname{sys}(X)}
$$

(since sys $(X) \geq 2 r_{0}$ ), where $v_{n}(r)$ denotes, as usual, the volume of a ball of radius $r$ in $\mathbb{H}^{n}$. Now notice that, as $k(X) \leq-1$, the diameter $d_{0}$ of $X_{0}$ can be bounded in terms of $n, r_{0}$ and $v_{0}$. Actually, if $\gamma_{0}$ is a minimizing geodesic of length $d_{0}$ between $x_{0}, y_{0} \in X_{0}$, then there exist at least $d_{0} /\left(r_{0} / 2\right)-1$ disjoint balls of radius $r_{0} / 4$ with centers $x_{i}$ on $\gamma_{0}$, and vol $B\left(x_{i}, r_{0} / 4\right) \geq v_{n}\left(r_{0} / 4\right)$ for all $i$; hence, $v_{0} \geq\left(2 d_{0} / r_{0}-1\right) v_{n}\left(r_{0} / 4\right)$ and $d_{0} \leq r_{0} v_{0} / v_{n}\left(r_{0} / 4\right)$. Thus, formula (17) can be expressed in the simpler form

$$
\Omega(X) \geq \frac{\log \left(1+c_{1}^{\text {sys }(X)}\right)}{c_{2} \cdot \operatorname{sys}(X)} \geq \frac{\log 2}{c_{2}} \cdot e^{-\left(\left|\log c_{1}\right|+1 / e\right) \cdot \operatorname{sys}(X)} \geq e^{-c \cdot \operatorname{sys}(X)}
$$

for suitable positive functions $c_{1}, c_{2}$ and $c$ of $n, R_{0}, v_{0}$. This proves Theorem 2 .

Proof of Proposition 22 The map $j$ is constructed by recurrence on the length of the sequence as follows. First, for every $\gamma \in \Gamma$ we fix a representative $g \in G$ which is minimal modulo $N$, ie $\|g\|=\|\gamma\|=d(x, \gamma(x))$. Then for a sequence of length 1 , 
that is a point $x_{1}=\gamma_{1} x \in S_{L}^{*}$, we pick the minimal representative $g_{1} \in G$ of $\gamma_{1}$ and we set $j\left(x_{1}\right)=g_{1}$; finally, for a general sequence of depth $m$, we set

$$
j\left(x_{1}, \ldots, x_{m}\right)=\left(g_{1}\right)_{\tau_{2} \ldots m} * \eta^{\kappa} * j\left(x_{2}, \ldots, x_{m}\right)
$$

where $\tau_{2 \ldots m}=\operatorname{sign} j\left(x_{2}, \ldots, x_{m}\right)$ and $\kappa \geq \kappa(L)$ as in Proposition 17.

It is clear that the map $j$ is contracting for our choice of $c$ as, by Proposition 11 and by (8)

$$
\begin{aligned}
\left\|j\left(x_{1}, \ldots, x_{m}\right)\right\| & \leq \sum_{i}\left\|g_{i}\right\|+(m-1)\left(\|\eta\|^{\kappa(L)}+3 \epsilon(L)\right) \\
& \leq \sum_{i} d\left(x, \gamma_{i}(x)\right)+7(m-1) \log \left(5 e^{4 L}+\operatorname{coth}(L / 2)\right) \\
& =\left\|\left(x_{1}, \ldots, x_{m}\right)\right\|_{c} .
\end{aligned}
$$

We must now show that the map $j$ is injective. Assume $j\left(x_{1}, \ldots, x_{m}\right)=j\left(\hat{x}_{1}, \ldots, \hat{x}_{\hat{m}}\right)$; this means that, if $x_{i}=\gamma_{i}(x), \hat{x}_{i}=\hat{\gamma}_{i}(x)$ and if $g_{i}, \hat{g}_{i}$ are the fixed representatives of $\gamma_{i}$ and $\hat{\gamma}_{i}$ which are minimal modulo $N$, we have

$$
\left(g_{1}\right)_{\tau_{2 \ldots m}} * \eta^{\kappa} * j\left(x_{2}, \ldots, x_{m}\right)=\left(\widehat{g}_{1}\right)_{\widehat{\tau}_{2 \ldots \hat{m}}} * \eta^{\kappa} * j\left(\widehat{x}_{2}, \ldots, \hat{x}_{\hat{m}}\right)
$$

where $\tau_{2 \ldots m}=\operatorname{sign} j\left(x_{2}, \ldots, x_{m}\right), \hat{\tau}_{2 \ldots \hat{m}}=\operatorname{sign} j\left(\hat{x}_{2}, \ldots, x_{\hat{m}}\right)$. Now, $\left(g_{1}\right)_{ \pm}$and $\left(\hat{g}_{1}\right)_{ \pm}$ are also $\epsilon(L)$-minimal modulo $\mathcal{N}_{G}(\eta)$, therefore Proposition 17 implies

$$
d\left(\gamma_{1}, \widehat{\gamma}_{1}\right) \leq d\left(\left(g_{1}\right)_{\tau_{2} \ldots m},\left(\widehat{g}_{1}\right)_{\widehat{\tau}_{2 \ldots \hat{m}}}\right) \leq \delta(L)
$$

but $\gamma_{1}, \hat{\gamma}_{1}$ belong to a $\delta(L)$-separated set, hence we deduce that $\gamma_{1}=\widehat{\gamma}_{1}$ and $g_{1}=\widehat{g}_{1}$ necessarily. Moreover, we deduce that $\tau_{2 \ldots m}=\hat{\tau}_{2 \ldots \hat{m}}$, otherwise

$$
d\left(\left(g_{1}\right)_{\tau_{2 \ldots m}},\left(\widehat{g}_{1}\right)_{\widehat{\tau}_{2 \ldots \hat{m}}}\right) \geq 2\left\|g_{1}\right\|-\epsilon(L)-\rho(L)>2 \delta(L)-\epsilon(L)-\rho(L)>\delta(L)
$$

by Propositions 11 and 12. We can then apply Proposition 15 twice (notice that $\eta^{k} * \beta$ always has the sign of $k)$ and infer that $\eta^{k} * j\left(x_{2}, \ldots, x_{m}\right)=\eta^{k} * j\left(\hat{x}_{2}, \ldots, \hat{x}_{m}\right)$ and that $j\left(x_{2}, \ldots, x_{m}\right)=j\left(\hat{x}_{2}, \ldots, \hat{x}_{\hat{m}}\right)$. We conclude therefore by induction that $m=\hat{m}$ and $x_{k}=\hat{x}_{k}$ for all $k$.

Proof of Corollary 5 Let $n=\operatorname{dim}\left(\mathcal{X}_{0}\right)$ and $r_{0}=\operatorname{inj}\left(\mathcal{X}_{0}\right)$. By Theorem 1 and a well-known formula of Brooks [2] we deduce

$$
\lambda_{0}(\mathcal{X}) \leq \frac{1}{4} \omega(\mathcal{X})^{2} \leq \frac{1}{4}\left(\omega_{\mathbf{K}, m}-e^{-c\left(n, r_{0}, v_{0}\right) \cdot \operatorname{sys}(\mathcal{X})}\right)^{2} \leq \lambda_{0}\left(\mathbf{K} \mathbb{H}^{m}\right)-\frac{1}{4} e^{-2 c\left(n, r_{0}, v_{0}\right) \cdot \operatorname{sys}(\mathcal{X})}
$$

because of Sullivan and Corlette's formula. By the same formula, we infer

$$
\omega(\Gamma)=\frac{1}{2} \omega_{\mathbf{K}, m}+\sqrt{\lambda_{0}\left(\mathbf{K} \mathbb{H}^{m}\right)-\lambda_{0}(\mathcal{X})} \geq \frac{1}{2}\left(\omega_{\mathbf{K}, m}+e^{-c\left(n, r_{0}, v_{0}\right) \cdot \operatorname{sys}(\mathcal{X})}\right) .
$$


Now $n=m \cdot \operatorname{dim}_{\mathbf{R}} \mathbf{K}$ and, as $k\left(\mathcal{X}_{0}\right) \geq-4$, Margulis' lemma gives $r_{0} \geq r_{0}(n)$; thus we can express $c\left(n, r_{0}, v_{0}\right)$ as a function $c_{\mathbf{K}, m}\left(v_{0}\right)$, which proves the corollary.

\section{References}

[1] G N Arzhantseva, I G Lysenok, Growth tightness for word hyperbolic groups, Math. Z. 241 (2002) 597-611 MR1938706

[2] R Brooks, A relation between growth and the spectrum of the Laplacian, Math. Z. 178 (1981) 501-508 MR638814

[3] M Brunnbauer, Homological invariants for asymptotic invariants and systolic inequalities, to appear in G.A.F.A. arXiv: math.GT/0702789

[4] K Corlette, Hausdorff dimensions of limit sets. I, Invent. Math. 102 (1990) 521-541 MR1074486

[5] E Ghys, P de la Harpe (editors), Sur les groupes hyperboliques d'après Mikhael Gromov, Progress in Mathematics 83, Birkhäuser, Boston (1990) MR1086648 Papers from the Swiss Seminar on Hyperbolic Groups held in Bern, 1988

[6] R Grigorchuk, P de la Harpe, On problems related to growth, entropy, and spectrum in group theory, J. Dynam. Control Systems 3 (1997) 51-89 MR1436550

[7] MG Katz, S Sabourau, Entropy of systolically extremal surfaces and asymptotic bounds, Ergodic Theory Dynam. Systems 25 (2005) 1209-1220 MR2158402

[8] G Reviron, Espaces de longueur d'entropie majorée: rigiditée topologique, adhérence des variétés, noyau de la chaleur, $\mathrm{PhD}$ thesis, l'Institut Fourier (2005)

[9] T Roblin, Un théorème de Fatou pour les densités conformes avec applications aux revêtements galoisiens en courbure négative, Israel J. Math. 147 (2005) 333-357 MR2166367

[10] A Sambusetti, Growth tightness of surface groups, Expo. Math. 20 (2002) 345-363 MR1940012

[11] D Sullivan, Related aspects of positivity in Riemannian geometry, J. Differential Geom. 25 (1987) 327-351 MR882827

Dipartimento di Matematica G Castelnuovo, Università "La Sapienza"

P.le Aldo Moro 5, 00185 Roma, Italy

sambuset@mat.uniroma1.it

http://www.mat.uniroma1.it/people/sambusetti/

Proposed: Jean-Pierre Otal

Received: 12 June 2006

Seconded: Benson Farb, David Gabai Accepted: 6 December 2007 